\title{
A continuous measure of object-based attention sheds new light on its underlying mechanisms
}

\section{Felipe Luzardo ${ }^{1}$, Wolfgang Einhäuser ${ }^{2} \&$ Yaffa Yeshurun ${ }^{1}$}

1

School of Psychological Sciences \& Institute of Information Processing and Decision

Making, University of Haifa; and ${ }^{2}$ Institute of Physics, Chemnitz University of Technology

\section{Corresponding Author:}

Felipe Luzardo, School of Psychological Sciences, University of Haifa, Haifa 3498838, Israel.

E-mail: felipedavidluzardo@gmail.com

\section{Acknowledgments}

This study was supported by the German-Israeli Foundation for Scientific Research and Development (GIF) Grant I-1418-105.4/2017. 


\begin{abstract}
Attention is largely guided by objects; when attending a location on an object, observers react faster to targets appearing in other locations within the object than outside the object. While crucial to our understanding of attention, there is no consensus on the mechanisms underlying such object-based attention. We describe a continuous measure of object-based attention that relies on attentional modulations of the pupillary light response (PLR). This response-free measurement provided unequivocal support for one of the debated accounts - 'attentional shifting', which underscores the higher cost of shifting attention between objects than within an object. In each trial, a precue appeared inside or outside a black/white object. The target followed in the same location as the cue or in a different location. In two experiments, changes in the PLR matched the shifting account's predictions, revealing slower attentional shifts when a disengagement was required (cue inside, target outside) than not required (cue outside, target inside).
\end{abstract}




\section{Introduction}

Object-based attention (OBA) refers to attentional processes whose unit of selection is an object. The assumption that attention can select objects has been consistently supported (reviewed in Chen, 2012). In a well-established demonstration of OBA (e.g., Egly, Driver \& Rafal, 1994), reaction times (RTs) to a task-defined target, appearing at a different location from an attentional cue, are faster when the target appears on the cued object than on a different object (even though target-cue distances are equal). Different mechanisms have been proposed for this object-based advantage, including the 'attentional shifting' account (e.g., Brown \& Denney, 2007; Lamy \& Egeth, 2002; Yeshurun \& Rashal, 2017).

The shifting of attention is a ubiquitous operation of the visual system that is composed of three different processes: disengaging, shifting, and engaging (Posner, 1980). Shifting attention from one object or location to another requires disengaging from the first before engaging the second. The shifting account proposes that object-based effects occur because when the cue appears inside an object and the target appears outside this object attention must first disengage from the initially attended object. This is a time-consuming process resulting in slower RTs than when disengagement from an object is not required, like when the cue and target appear at different locations within the same object. Using one-object displays, Brown and Denney (2007) showed that RTs were longer when a trial involved disengaging attention from a cued object than when it did not. For instance, RTs were significantly slower when the cue was inside the object and the target was outside the object than in any other condition (i.e., cue outside object and target inside, cue and target both outside, or cue and target both inside). 
This was taken as evidence that disengaging attention from an object is a critical mechanism of OBA.

Although OBA has been studied extensively (Chen, 2012; Reppa et al., 2012), there is still no agreement regarding its underlying mechanisms (e.g., Nah \& Shomstein, 2020). Some question whether OBA can truly be attributed to attentional shifting (e.g., Drummond \& Shomstein, 2010; Luo \& Proctor, 2016), and alternative mechanisms have been suggested, like 'attentional spreading' - the automatic spreading of attention from the cued location to other locations within the cued object (e.g., Chen \& Cave, 2006; Richard et al., 2008) or 'attentional prioritization' - the assignment of higher attentional priority to locations within the attended object (e.g., Drummond \& Shomstein, 2010; Shomstein \& Yantis, 2002). Here, we used the attentional modulation of the pupillary light response (PLR) to reexamine whether object-based effects can be attributed at least partially to attentional shifting.

It is well-known that cognitive effort and arousal can lead to pupillary dilation (reviewed in Einhäuser, 2017). But the PLR was largely regarded as a low-level reflex that controls pupil size to adapt to incident light levels, increasing/decreasing pupils' size in dark/bright light levels (e.g., Loewenfeld, 1999). Recent studies, however, have shown that high-level processes also affect the PLR (e.g., Laeng \& Endestad, 2012; Naber \& Nakayama, 2013). Importantly, covert attention (i.e., attention allocation in the absence of eye movements) can modulate the PLR. Covertly shifting spatial attention to a brighter area produces pupillary constriction, relative to shifting attention to a darker area, under identical amounts of luminance, arousal, and cognitive load (e.g., Binda et al., 2013; Mathôt et al., 2013, 2014; Naber et al., 2013; Tkacz-Domb \& Yeshurun, 
2018). Therefore, changes in the PLR can be used to infer how attention is allocated over the course of a trial. Using the PLR avoids unintended contamination of performance by factors that are unrelated to attention allocation or beyond experimental control. Critically, the attentional modulation of the PLR is a temporally continuous measure that allows tracking the effect of attention throughout a trial. This is critical for assessing the involvement of disengaging processes as postulated by the shifting account of OBA. By continuously tracking the effect of attention on the PLR one can assess whether switches of attention that involve disengaging from an object are indeed slower than those that do not involve such disengagement because this should be reflected in slower changes elicited by the PLR.

To that end, we employed single-object displays, similar to Brown and Denney (2007), but the object was either black or white. We used differences in the PLR to infer the time-course of attentional deployment throughout different conditions. The critical comparison was between trials in which the cue appeared inside the object followed by a target outside the object and trials in which the cue appeared outside the object followed by a target inside. The former involved disengaging attention from the object while the latter did not. If disengaging attention from an object is indeed a particularly time-consuming process that slows down attentional shifting, changes in the PLR should be observed later in the first case than in the second case. In Experiment 2 we increased the luminance of stimuli when they appeared on the gray background (outside the object). This was done to rule out the higher saliency of stimuli on the white/black background (inside the object) as an alternative explanation for the findings of Experiment 1. 


\section{Experiment 1}

\section{Method}

Participants. Because the effects of OBA were not studied before with the PLR, we had no obvious prior work on which to base a power analysis. However, PLR effect sizes (Cohen's d) with space-based attention are slightly larger than 1 (Mathôt et al., 2013). A power analysis (using the R pwr package; Champely, 2020) indicated that a sample size of 15 is necessary for a power of 0.95 (alpha $=0.05, d=1$ ). The sizes of OBA effects are typically smaller than those of space-based attention (e.g., Egly, Driver \& Rafal, 1994; Yeshurun \& Rashal, 2017), and we therefore aimed for at least 20 participants in each of our experiments. In the end, due to the recruitment procedure, 23 students from the University of Haifa participated in this experiment. All participants reported normal or corrected to normal vision and no history of neurological disorders, and all signed a consent form. One participant was excluded due to a high percentage of trials with eye movements (above $20 \%$ ). The study was approved by the ethics committee of the University of Haifa (approval number 265/17).

Stimuli and apparatus. Stimuli were generated and presented using Psychopy (Peirce et al., 2019) on a 19-in. CRT monitor $(1,280 \times 1024$ resolution at a refresh rate of 85 $\mathrm{Hz}$ ). Eye movements and pupil size were recorded from the right eye with an EyeLink 1000 eye tracker (temporal resolution of $1000 \mathrm{~Hz}$; SR Research, Ottawa, ON, Canada). Stimuli were presented on a gray background $\left(14 \mathrm{~cd} / \mathrm{m}^{2}\right)$. The object was a bright $(80$ $\left.\mathrm{cd} / \mathrm{m}^{2}\right)$ or dark $\left(0 \mathrm{~cd} / \mathrm{m}^{2}\right)$ vertical rectangle $\left(4^{\circ} \times 12^{\circ}\right)$ with a red outline presented at an eccentricity of $5^{\circ}$ (measured from the center of the screen to the center of the object; Fig. 1a). The fixation mark was a red cross $\left(1^{\circ} \times 1^{\circ}\right)$. The cue was a red circle (diameter: 
$1^{\circ}$ ) appearing at one of four locations (inside or outside the object; Fig. 1a) at an eccentricity of $7^{\circ}$. The target was a red letter (' $\mathrm{Z}$ ' or ' $N$ '; $1^{\circ}$ height) presented at one of the four locations. All red stimuli were isoluminant to the gray background $\left(14 \mathrm{~cd} / \mathrm{m}^{2}\right)$.

Procedure. A 5-point calibration preceded a practice session, and additional calibrations were performed as needed. The participants were instructed to fixate the center of the screen throughout all trials. A trial started only after observers fixated the center of the screen; if needed, the eye-tracker was recalibrated. Each trial began with the fixation cross and the object (Fig. 1b). The object was dark in half of the trials and light in the other half. After 1 second, the cue appeared for $150 \mathrm{~ms}$, and following a 150ms interstimulus interval (ISI) the target was displayed until response. The cue had an equal probability of appearing in any of the four possible locations and it correctly predicted the location of the target in $60 \%$ of trials (valid trials). In the remaining $40 \%$ of trials (invalid trials), the target always appeared on the other side of the display (outside the object if the cue had been inside, and inside the object if the cue had been outside; Fig. 1b). The participants had to indicate if the target was a ' $Z$ ' or an ' $N$ '. Auditory feedback followed the participants' response. If the eye-tracker detected an eye position that was more than $1.5^{\circ}$ away from the center of the display during a trial, the trial was immediately canceled, and auditory feedback was given to indicate that eye-movements were detected. Participants completed 30 practice trials and 480 experimental trials ( 45 minutes). Trial types (i.e., object's location and luminance, target/cue position) were mixed randomly while keeping the same number of trials in each condition for every participant. 
a

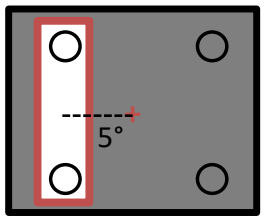

b

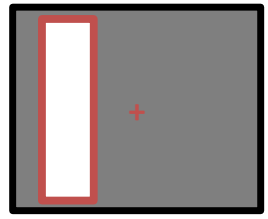

$1 \mathrm{sec}$

fixation

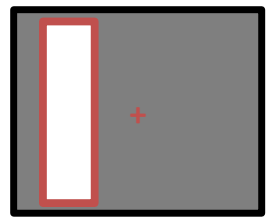

$1 \mathrm{sec}$
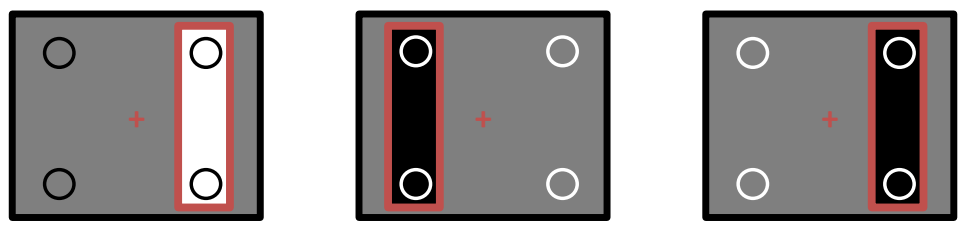

target

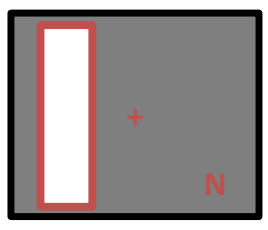

invalid

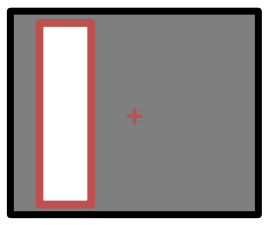

$150 \mathrm{~ms}$

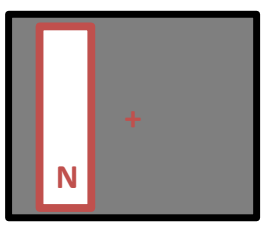

until response

target

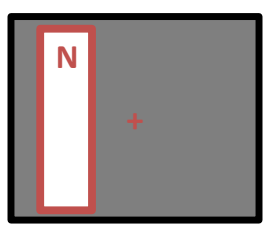

invalid

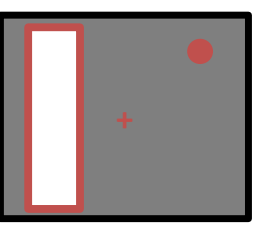

$150 \mathrm{~ms}$
ISI

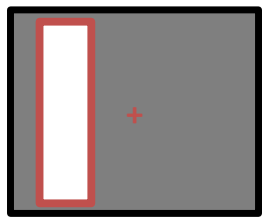

$150 \mathrm{~ms}$

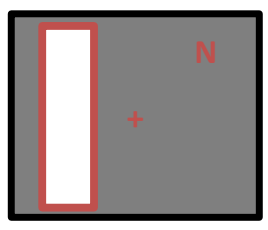

valid

until response

Fig. 1. (a) All possible stimuli locations and luminance levels in Experiment 1. The circles demonstrate the possible target and cue locations, but they were not included in the actual task. (b) Stimuli and sequence of events for the different types of trials. A single rectangle was presented to the left or right of fixation, the rectangle could be white or black. A circle cue was briefly displayed inside or outside the object, and a target ("N" or "Z") followed either at the same location (valid condition) or the other location (invalid condition). Figure not to scale.

Pupil size analysis. Trials that were canceled because of eye movements were removed from all analyses (see Procedure; $9.53 \%$ of total trials). Pre-processing of pupil 
size data was done in $\mathrm{R}$ using the gaze $R$ package (Geller et al., 2020). Blink events were linearly interpolated (Bradley et al., 2008; Tkacz-Domb \& Yeshurun, 2018; Wolff et al., 2015). Then, the median absolute deviation was used to detect rapid pupil-size disturbances in the data (Kret \& Sjak-Shie, 2018). Such data points were removed ( $0.25 \%$ of total data points). The data was then smoothed using a 5-point moving average (e.g., Schmidtke, 2018; Zekveld \& Kramer, 2014). The baseline was the median pupil size of the $100 \mathrm{~ms}$ period preceding the onset of the rectangle. For each trial, the pupil size at each time point was divided by the baseline value.

Pupil-size difference. Following pre-processing, the pupil-size difference was calculated by subtracting average pupil size in light-object trials from average pupil size in darkobject trials. This difference was calculated separately for each time point, validity condition (valid/invalid), and cue location condition (inside-object/outside-object), but averaged over stimuli locations. Linear mixed-effects analyses (LME) were performed for each time point in $\mathrm{R}$ using the Ime4 package (Bates et al., 2014). The pupil-size difference was entered as the dependent variable and subject ID as a random effect. To evaluate the time course of the effect of validity differentially for the different cue location conditions, we ran separate LMEs for each cue location condition with validity as a fixed effect. Similar to previous studies, the significance criterion was defined as t>2 for at least 200 consecutive ms (e.g., Mathôt et al., 2013; Mathôt, et al., 2014; Tkacz-Domb \& Yeshurun, 2018).

Cueing difference. To directly compare shifting latencies between the cue-inside and cue-outside conditions, a 'cueing difference' measurement was calculated by subtracting pupil-size difference in the invalid condition from that in the valid condition 
separately for each time point, participant, and cue location. Because the valid and invalid trials were identical up to the target onset, any cueing difference up to this point can be attributed to random noise. Additionally, it takes a relatively long time for the pupil to react to stimuli, especially with low-intensity stimuli such as the target (Ellis, 1981). Therefore, to reduce the effect of random noise, the cueing difference value observed $300 \mathrm{~ms}$ following target onset was considered as the baseline noise and was subtracted from all cueing difference values, separately for each time point, participant, and cue location. Additionally, a cueing difference threshold was determined as 2 standard deviations (SD) of the random noise (the cueing difference in a $1300 \mathrm{~ms}$ window from object onset up to $300 \mathrm{~ms}$ following target onset), individually for each participant and each cue location. Note that restricting the random noise window to the $300 \mathrm{~ms}$ following target onset for the purposes of threshold calculation would not have changed the conclusions of the analysis. Similarly, calculating a single threshold for all participants rather than individually, would not have changed the conclusions of the analysis. We then marked, for each individual and each cue location, the time point from $300 \mathrm{~ms}$ and onward at which the cueing difference crossed the threshold. This time point, at which the cueing difference became greater than random noise, was taken as the time point of an attentional shift and was entered as the dependent variable in a paired t-test. One participant's cueing difference did not reach the threshold at all in at least one condition and was therefore removed from the analysis.

Behavioral analysis. Accuracy in the task was near ceiling (97.83\%), therefore accuracy is not discussed further. Trials with incorrect responses were removed $(2.17 \%$ of trials) prior to analyzing RT data. Responses faster or slower than 2 SD from the 
mean (calculated separately for each participant) were also removed (3.84\% of trials) from the RT analysis. The data was analyzed in a two-way repeated-measures analysis of variance (ANOVA) that included cue validity (valid/invalid) and cue location (insideobject/outside-object) as independent variables and RT as the dependent variable. Target saliency. Because the target contrast was larger when it appeared on the white/black background than on the gray background, we conducted a one-way repeated measures ANOVA including only valid trials to examine the effect of target saliency levels as a function of the three different backgrounds. We examined only valid trials here to focus on the effect of target saliency without the influence of attention shifting. Target background (white/black/gray) was the independent variable and RT was the dependent variable.

\section{Results}

Pupil size difference. When the cue appeared inside the object, pupil-size difference initially increased reflecting the fact that attention was directed towards the object (i.e., towards a region whose luminance varied considerably between light/dark trials; Fig. 2a). Conversely, when the cue appeared outside the object, pupil-size difference initially decreased reflecting the fact that attention was directed towards the gray background (i.e., towards a region whose luminance did not vary between light/dark trials). At first, this pattern of results was similar for the valid and invalid trials. This is expected given the fact that up until target onset the valid and invalid trials were identical. However, about $400 \mathrm{~ms}$ following target onset, which is the typical time required for the pupil to 'react' to an event (Ellis, 1981), the pattern of results observed with the valid trials started to differ from invalid trials. Specifically, in the valid trials, the same pattern of 
results as described above remained because no shift of attention was needed. In contrast, in the invalid trials, attention had to shift from the cued location to the target location, and the luminance levels at the cue and target locations were always different. Therefore, a change in the pattern of attentional modulation of the PLR was expected. Indeed, following target onset we see a switch in the pattern of results for the invalid condition: A decreasing pupil-size difference with the inside-object condition, reflecting the attentional shift from the cued location inside the object towards the target location outside the object, and an increasing pupil-size difference with the outside-object condition, reflecting the attentional shift from the cued location outside the object towards the target location inside the object. Importantly, a significant difference between the pattern of results of the valid and invalid trials emerged later when the cued location was inside the object (419ms; Fig. $2 b)$ than when it was outside the object (409ms). 

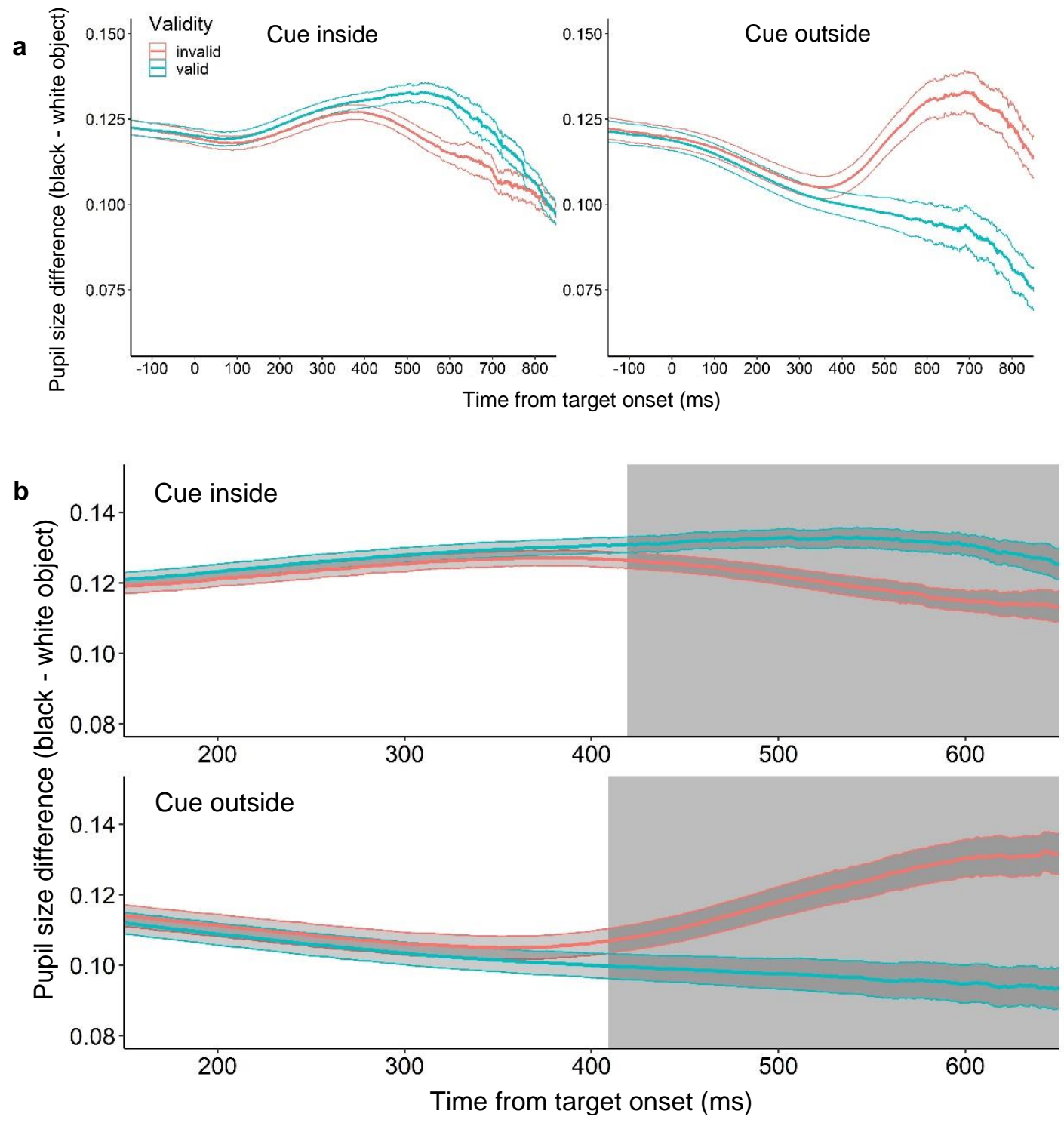

Fig. 2. Results of Experiment 1. a. Overview of pupil-size difference (calculated by subtracting pupil size in light-object trials from pupil size in dark-object trials) as a function of time from target onset. The baseline was the median pupil size of the last $100 \mathrm{~ms}$ before object onset. b. Zoomed-in view of the critical period in the experiment. The gray shading indicates a significant difference between the valid and invalid conditions, which emerged earlier in the cue outside-object condition. The area around the curves represents one standard error of the mean. 
Cueing difference. To directly compare shifting latencies between the cue location conditions (inside/outside), the 'cueing difference' was calculated by subtracting individual pupil-size difference in the invalid condition from the valid condition for each cue location (see Methods; Fig. 3a). Then, the time at which the cueing difference became greater than random noise was taken as the shifting time (Fig. 3b). The shifting time was significantly greater for the cue-inside (mean $=513 \mathrm{~ms}, 95 \% \mathrm{Cl}=[406,756])$ than the cue-outside condition (mean $=456 \mathrm{~ms}, 95 \% \mathrm{Cl}=[382,536] ; t(20)=2.93, p=.008$; Fig 3c). This suggests that the shift of attention started later in the former (inside-to-outside) than the latter (outside-to-inside) condition, as expected if the disengagement from the cued location is slower (or costlier) when it is inside the object. This is in line with the shifting account of OBA that predicts slower shifts of attention when a disengagement from an object is required.

Behavioral data. A significant main effect of validity was revealed $(F(1,21)=108.49$, $p<.001, \eta_{p}^{2}=.63$; Table 1). The main effect of cue location was not significant $\left(F(1,21)=3.61, p=.062, \eta_{p}^{2}=.05\right)$. In line with the shifting account, we found a significant interaction between cue location and validity $(F(1,21)=96.15, p<.001$, $\eta_{p}^{2}=.60$ ). A preplanned comparison (paired t-test) revealed significantly faster RTs for invalid trials when the cue appeared outside than inside the object $(t(21)=5.54, p<.001$; Table 1). This is consistent with the postulation that attentional shifts are slower when they involve disengaging from an object than when they do not. 

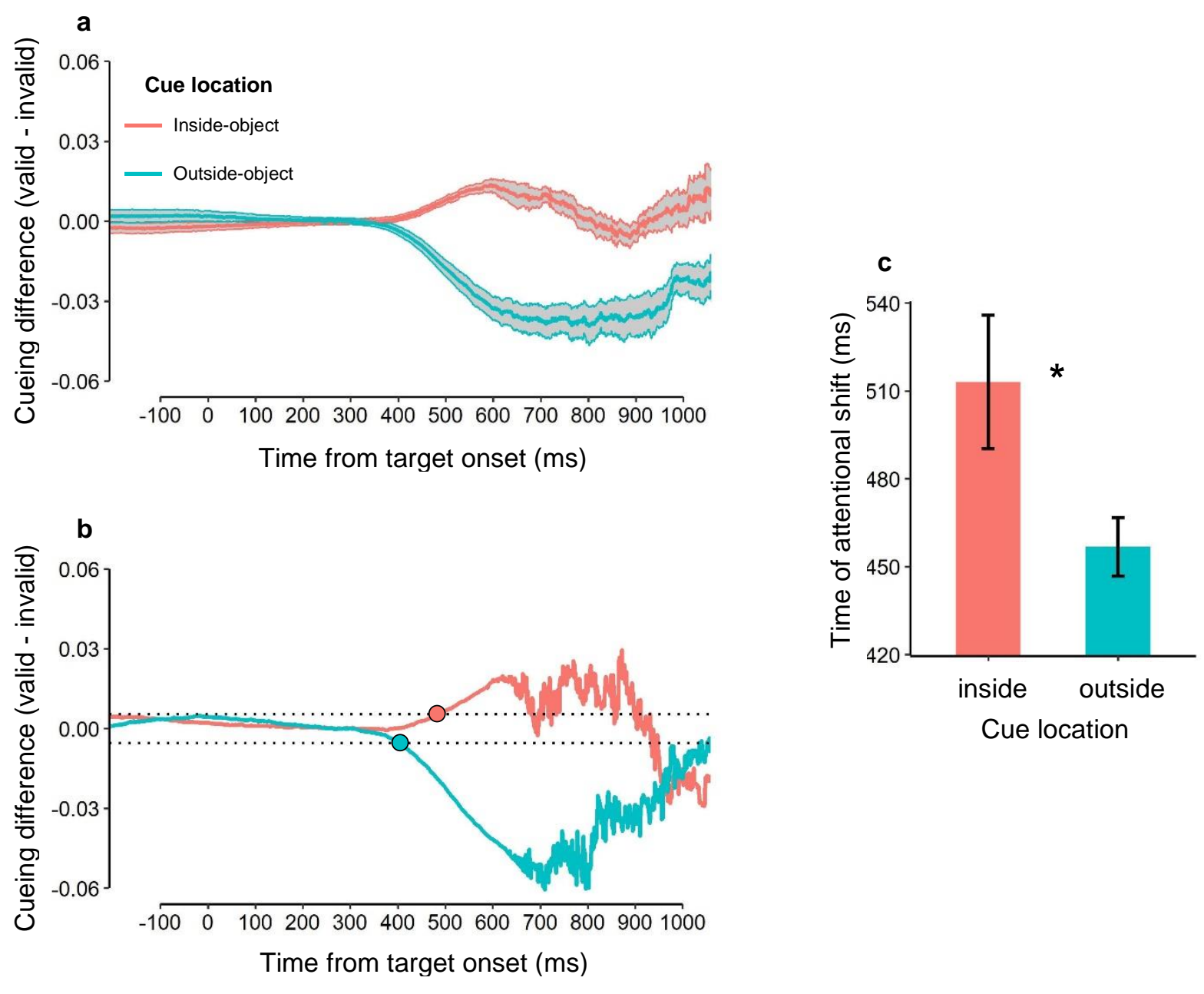

Fig 3. Cueing difference in Experiment 1. a. Mean cueing difference (calculated by subtracting pupil-size difference in the invalid condition from that of the valid condition) as a function of time. The shaded area around each curve denotes one within-subject standard error (Cousineau, 2005). b. Example cueing difference from a single participant. Dotted horizontal lines represent this participant's individual threshold (see Method section for details). The time point at which the cueing difference reached this threshold for each cue location condition (indicated by a dot) was taken as the time of attentional shift for each participant. c. Mean time point of attentional shift in each cue location condition. Error bars represent one within-subject standard error.

Target saliency. A significant effect of target background was revealed $(F(1,21)=49.78$, $\left.p<.001, \eta_{p}^{2}=.70\right)$. Follow-up comparisons revealed a significant difference $(t(21)=8.77$, 
$p<.001)$ between the white $($ mean $=464 \mathrm{~ms}, 95 \% \mathrm{Cl}=[438,490])$ and gray (mean=497ms, $95 \% \mathrm{Cl}=[471,522])$ backgrounds and between the black (mean=472ms, 95\% $\mathrm{Cl}=[448$, 497]) and gray backgrounds $(t(21)=6.47, p<.001)$. The slower response to targets appearing on the gray background indicates that the targets were less salient when appearing on the gray background (outside-object) than on the black/white background (inside-object). This salience difference might have enabled participants to detect the target faster inside than outside the object. Such speed differences in target detection could partially explain the pattern of PLR results because in the invalid outside-object condition the target appeared inside the object, and if it was detected faster this could potentially lead to an earlier attentional shift in comparison to the invalid inside-object condition. To rule out this alternative explanation, we attenuated in Experiment 2 the salience differences between targets appearing on different backgrounds. Finally, there was a small but significant difference between the white and black backgrounds $(t(21)=$ 3.26, $p=.011$ ) suggesting that the target was slightly easier to detect on the white background.

Table 1. Mean reaction times for the different conditions in Experiment $1(N=22)$

\begin{tabular}{llcc}
\hline Cue location & Validity & Reaction time $(\mathrm{ms})$ & $95 \% \mathrm{Cl}$ \\
\hline inside-object & invalid & 518 & {$[514,523]$} \\
inside-object & valid & 468 & {$[465,471]$} \\
outside-object & invalid & 497 & {$[492,501]$} \\
outside-object & valid & 496 & {$[493,500]$} \\
\hline
\end{tabular}




\section{Experiment 2}

\section{Method}

Participants. Twenty-two participants from the University of Haifa participated in this experiment after signing a consent form. All participants reported normal or corrected to normal vision and no history of neurological disorders.

Stimuli apparatus and procedure. The stimuli apparatus and procedure were identical to Experiment 1 except for the following changes. Stimuli that were presented in red in Experiment 1 were presented in green $\left(14 \mathrm{~cd} / \mathrm{m}^{2}\right)$. When the cue or the target appeared on a grey background (outside the object), their luminance was increased to $38 \mathrm{~cd} / \mathrm{m}^{2}$ to increase stimulus salience in these cases, bringing it up to par with stimulus salience on the white or black backgrounds (inside the object). This value was chosen based on a short pilot study, which demonstrated that with this luminance modification target identification on the gray background is comparable to that on the white or black backgrounds and is further validated by the analysis of the valid conditions of this experiment (see Results section).

Pupil size analysis. Pre-processing and statistical analyses were identical to those employed in Experiment 1 (9.85\% of total trials removed due to eye movements). Three participants' cueing differences did not reach their respective thresholds at all in at least one condition and were therefore removed from the analysis.

Behavioral analysis. As in Experiment 1, accuracy in the task was near ceiling $(96.83 \%)$, and it is not discussed further. Trials with incorrect responses (3.17\% of trials) or responses that are too fast/slow (same exclusion criterion as in Experiment $1 ; 4.22 \%$ 
of trials) were removed from the RT analysis. We performed a similar ANOVA on RT as in Experiment 1, and then we conducted another ANOVA only on valid trials to examine target saliency on the different backgrounds.

\section{Results}

Pupil size difference. A similar pattern to that seen in Experiment 1 emerged here for the pupil-size difference in all conditions (Fig. 4a). Importantly, as in Experiment 1, a significant difference in the pattern of results (i.e., whether the pupil-size difference increases or decreases) between the valid and invalid conditions emerged earlier for the outside-object condition (358ms; Fig. 4b) than the inside-object condition (483ms).

Cueing Difference. A similar pattern to Experiment 1 was also observed here (Fig. 5a). The shifting time was significantly later in the cue inside (mean $=585,95 \% \mathrm{Cl}=[422,923]$ ) than the cue outside condition ( mean $=455,95 \% \mathrm{Cl}=[375,525] ; t(18)=4.39, p<.001$; Fig. 5b). Because here the target salience was similar in these two conditions (see analyses below), the finding of an earlier shifting time in the outside-object condition strengthens the conclusion that shifts of attention are indeed slower when a disengagement from an object is required, as suggested by the shifting account of OBA. 

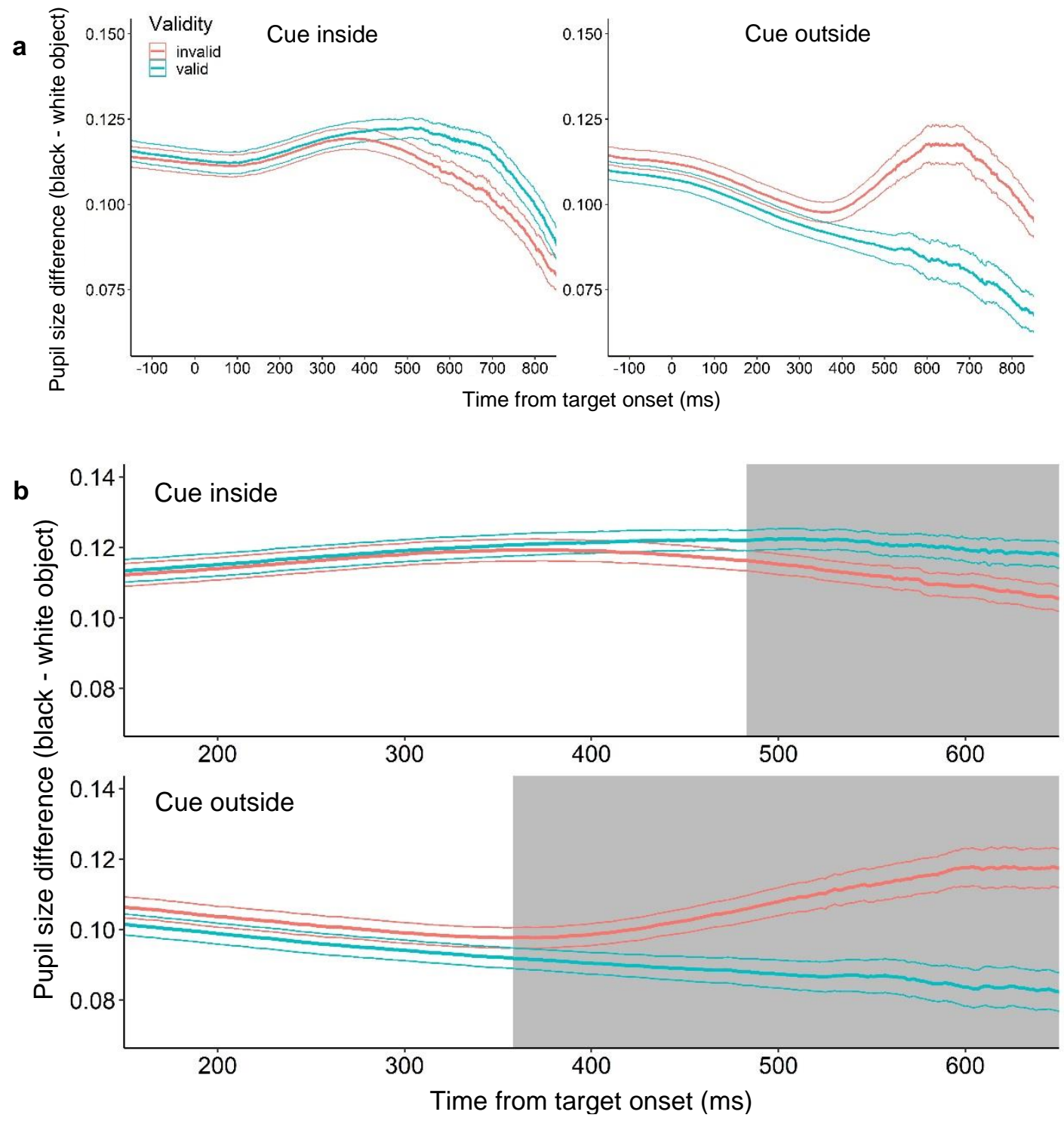

Fig. 4. Results of Experiment 2. a. Overview of pupil-size difference (calculated by subtracting pupil size in light-object trials from pupil size in dark-object trials) as a function of time from target onset. The baseline was the median pupil size of the last $100 \mathrm{~ms}$ before object onset. $\mathrm{b}$. Zoomed-in view of the critical period in the experiment. The gray shading indicates a significant difference between the valid and invalid conditions, which occurred earlier in the cue outside-object condition. The area around the curves represents one standard error of the mean. 
Behavioral data. A significant main effect of validity emerged $(F(1,21)=81.89, p<.001$, $\eta_{p}^{2}=.57$; Table 2). The main effect of cue location $\left(F(1,21)=0, p=.98, \eta_{p}^{2}=0\right)$ and the interaction $\left(F(1,21)=.04, p=0.85, \eta_{p}^{2}=0\right)$ were not significant.

Target saliency. A significant effect of target background was revealed $(F(1,21)=7.20$, $\left.p=.002, \eta_{p}^{2}=.26\right)$. However, this significant effect was not due to differences between the gray and white/black backgrounds because there was no significant difference $(t(21)=2.03, p=.16)$ between trials with a gray background $($ mean $=464 \mathrm{~ms}, 95 \%$ $\mathrm{Cl}=[440,489])$ and trials with a white $(\mathrm{mean}=459 \mathrm{~ms}, 95 \% \mathrm{Cl}=[435,484])$ or black (mean=469ms, 95\% Cl=[443,494]; $t(21)=1.75, p=.28$ ) background. This finding indicates that the targets' salience was similar on the gray background (outside-object) and the black/white background (inside-object), and therefore, salience differences cannot account for the different time-course of PLR dynamics observed with the inside-object and outside-object conditions. The significant effect of background reported above is due to significantly slower RTs $(t(21)=3.82, p=.003)$ on trials with a black than a white background. It is conceivable that these slower responses contributed to the lack of a significant cue location $\mathrm{x}$ validity interaction reported in the previous section, but in any case, they cannot account for the PLR results. 

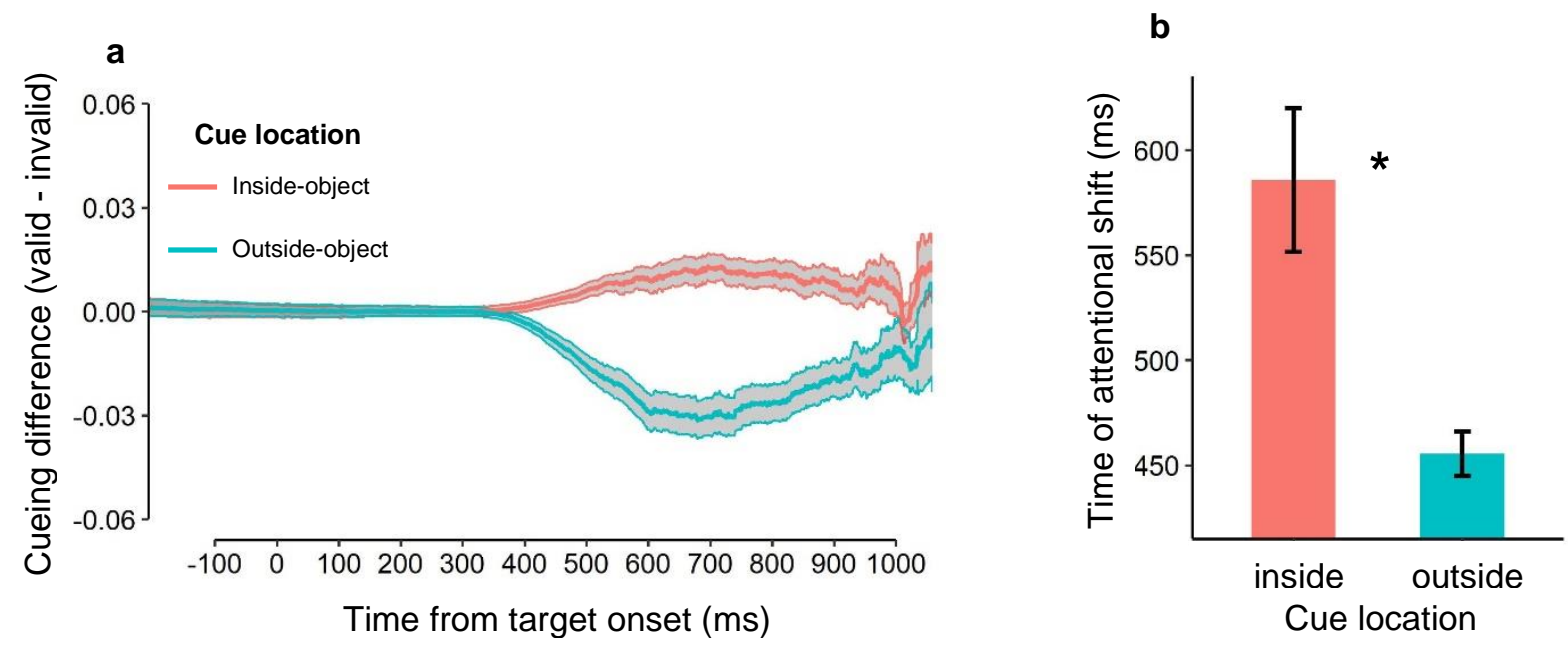

Fig. 5. Cueing difference in Experiment 2. a. Mean cueing difference (calculated by subtracting pupil-size difference in the invalid condition from that of the valid condition) as a function of time. The shaded area around each curve denotes one within-subject standard error. b. Mean time point of attentional shift in each cue location condition. Error bars represent one within-subject standard error.

Table 2. Mean reaction times for the different conditions in Experiment $2(N=22)$

\begin{tabular}{llcc}
\hline Cue location & Validity & Reaction time $(\mathrm{ms})$ & $95 \% \mathrm{Cl}$ \\
\hline inside-object & invalid & 482 & {$[477,486]$} \\
inside-object & valid & 463 & {$[460,466]$} \\
outside-object & invalid & 481 & {$[477,485]$} \\
outside-object & valid & 465 & {$[461,468]$} \\
\hline
\end{tabular}




\section{Discussion}

Most OBA studies relied on behavioral responses such as $\mathrm{RT}$ and were unable to produce unequivocal evidence for its underlying mechanisms. We took advantage of the attentional modulation of the PLR while participants performed a task that involved shifting attention from an object to a location or vice versa. It has been consistently shown that the PLR is a reliable measure of the deployment of covert attention (e.g., Binda et al., 2013; Mathôt et al., 2013; Naber et al., 2013; Yeshurun, 2019). Indeed, researchers have utilized the PLR to characterize different aspects of attentional allocation, like the size of the attentional window (Tkacz-Domb \& Yeshurun, 2018) or the focus of attention during spatial working memory tasks (Fabius et al., 2017). This study is the first to demonstrate that the PLR can also be used to characterize OBA. Specifically, we took advantage of the time-continuous nature of pupil size readings which is particularly important when examining the shifting account of OBA because its principal prediction is that attentional shifts that involve disengaging from an object are slower than those that do not. In two experiments, pupil-size differences consistently matched the shifting account's predictions, showing slower changes in the attentional modulation of the PLR in the invalid condition when the cue appeared inside the object (i.e., the attentional shift required disengaging from the object) compared to when the cue appeared outside the object (i.e., the attentional shift did not involve disengagement from the object). This pattern of results was observed regardless of saliency differences suggesting that it indeed reflects slower attention shifts when a disengagement from an object is required. 
Our findings add to the growing body of evidence demonstrating the role of attentional shifting in OBA (e.g., Brown \& Denney, 2007; Ho \& Atchley, 2008; Lamy \& Egeth, 2002; Yeshurun \& Rashal, 2017). Lamy and Egeth (2002), for example, measured RT in a size discrimination task and found object-based effects only when a shift of attention between objects was required, but not when attention was broadly distributed or when it could remain focused on a specific location. Yeshurun and Rashal (2017) employed a similar display and procedure to that of Egly et al. (1994) but added a neutral condition in which the precue did not indicate a location. They found the typical OBA effect faster RTs when the cue and target appeared within the same object (invalid-same condition) than when they appeared on different objects (invalid-different condition). Yet faster RTs were found in the neutral than invalid-same condition. This finding is consistent with the shifting account because, unlike other accounts of OBA, with the shifting account the invalid-same condition has no advantage over the neutral condition; in both conditions there is no need to disengage from an object. Still, the neutral condition has an advantage over the invalid-same condition because unlike the latter it also does not require disengaging from a location. Importantly, in contrast to previous studies which relied on the participants' response at the end of the trials, the current study provides a continuous measure of how attention is deployed within the course of a trial, and therefore it provides the most direct evidence for the attentional shifting account.

The general time-course in the black-white pupil-size differences was qualitatively similar across experiments. Nonetheless, the difference between the time-points of valid-invalid separation in the "cue-inside object" compared to the "cue-outside object" 
conditions was considerably larger in Experiment 2. We speculate that this may be related to the target/cue salience because it was the only methodological difference between the two experiments (apart from the individual differences between the participants of the two experiments). Conceivably, in Experiment 1, salience differences between conditions in which the target/cue appeared on a background of different luminance (i.e., inside the object vs. outside the object) have also affected the pattern of attention allocation, thereby diluting the effects of OBA. Importantly, because in Experiment 2 the target/cue salience was approximately matched between the insideobject and outside-object conditions, salience differences cannot account for the large differences in PLR observed in this experiment.

A single object in a visual scene can capture attention automatically (e.g., Kimchi et al. 2007). Therefore, it is conceivable that the single object in this study's displays initially captured attention. However, all pupil size analyses presented here were done within the same cue location condition. The valid and invalid trials within the same cue location condition were identical before target onset. Therefore, differences between valid and invalid trials that emerge after target onset are not affected by such initial capture of attention.

It is important to note that we do not rule out the possibility that other mechanisms such as attentional spreading or attentional prioritization also contribute to OBA under different circumstances. However, given the stimuli and procedure of this study, other accounts cannot explain the current findings. For instance, both the attentional spreading and attentional prioritization accounts were introduced to explain the faster RTs observed for the invalid-same than invalid-different condition. Yet in our study there 
is no invalid-same condition, and therefore spreading attention along the object or assigning higher priority to uncued locations within the cued object cannot account for the faster change in the pattern of PLR observed for the invalid-outside-object condition than invalid-inside-object condition. Additionally, it was suggested that OBA effects depend, at least partially, on whether the attentional shift crossed interhemispheric or intrahemispheric boundaries (e.g., Barnas \& Greenberg, 2019). However, in the current study, both invalid conditions (inside-object and outside-object) required the crossing of the interhemispheric boundary, and therefore this crossing cannot explain the observed PLR differences between these conditions. Finally, Chen and colleagues (2020) suggested that several demonstrations of OBA effects reflect the complexity of the regions between the cue and target in terms of object boundaries. This alternative explanation cannot account for our findings because with both invalid-inside-object and invalid-outside-object conditions the cue and target were separated by an object boundary. Thus, although all these different accounts of OBA may certainly be relevant with different stimuli and procedures, they cannot account for the findings of the current study.

In conclusion, this study shows that the measurement of the attentional modulations of the PLR can be used successfully in the study of OBA. Furthermore, we provide direct evidence in favor of the shifting account of OBA. 


\section{Declarations}

Funding. This study was supported by the German-Israeli Foundation for

Scientific Research and Development (GIF) Grant I-1418-105.4/2017.

Conflicts of interest. None to report.

Ethics approval. This study was performed in line with the principles of the Declaration of Helsinki. Approval was granted by the ethics committee of the University of Haifa (approval number 265/17).

Consent to participate. Informed consent was obtained from all individual participants included in the study.

Consent for publication. Not applicable.

Data availability. Data for all experiments have been made publicly available on OSF at: https://osf.io/d7sxe/?view_only=e5b3e8cd844e457db208b2c272faefc4

The design and analysis plans for the experiments were not preregistered.

Code availability. Not applicable. 


\section{References}

Barnas, A. J., \& Greenberg, A. S. (2019). Object-based attention shifts are driven by target location, not object placement. Visual Cognition, 27(9-10), 768-791.

Bates, D., Mächler, M., Bolker, B., \& Walker, S. (2014). Fitting linear mixed-effects models using Ime4. ArXiv Preprint ArXiv:1406.5823.

Binda, P., Pereverzeva, M., \& Murray, S. O. (2013). Attention to bright surfaces enhances the pupillary light reflex. Journal of Neuroscience, 33(5), 2199-2204.

Bradley, M. M., Miccoli, L., Escrig, M. A., \& Lang, P. J. (2008). The pupil as a measure of emotional arousal and autonomic activation. Psychophysiology, 45(4), 602-607.

Brown, J. M., \& Denney, H. I. (2007). Shifting attention into and out of objects: Evaluating the processes underlying the object advantage. Perception \& Psychophysics, 69(4), $606-618$.

Champely, S. (2020). pwr: Basic Functions for Power Analysis. R package version 1.3-0. https://CRAN.R-project.org/package=pwr

Chen, Z. (2012). Object-based attention: A tutorial review. Attention, Perception, \& Psychophysics, 74(5), 784-802.

Chen, Z., \& Cave, K. R. (2006). Reinstating object-based attention under positional certainty: The importance of subjective parsing. Perception \& Psychophysics, 68(6), $992-1003$.

Chen, Z., Cave, K. R., Basu, D., Suresh, S., \& Wiltshire, J. (2020). A region complexity effect masquerading as object-based attention. Journal of Vision, 20(7):24, 1-15. 
Cousineau, D. (2005). Confidence intervals in within-subject designs: A simpler solution to Loftus and Masson's method. Tutorials in quantitative methods for psychology, 1(1), 4245.

Drummond, L., \& Shomstein, S. (2010). Object-based attention: Shifting or uncertainty? Attention, Perception, \& Psychophysics, 72(7), 1743-1755. https://doi.org/10.3758/APP.72.7.1743

Egly, R., Driver, J., \& Rafal, R. D. (1994). Shifting visual attention between objects and locations: Evidence from normal and parietal lesion subjects. Journal of Experimental Psychology: General, 123(2), 161-177.

Einhäuser, W. (2017). The pupil as marker of cognitive processes. In Computational and cognitive neuroscience of vision (pp. 141-169). Springer.

Ellis, C. J. (1981). The pupillary light reflex in normal subjects. British Journal of Ophthalmology, 65, 754-759.

Fabius, J., Mathôt, S., Schut, M., Nijboer, T., \& Van der Stigchel, S. (2017). Focus of spatial attention during spatial working memory maintenance: Evidence from pupillary light response. Visual Cognition, 25(1-3), 10-20.

Geller, J., Winn, M. B., Mahr, T., \& Mirman, D. (2020). GazeR: A package for processing gaze position and pupil size data. Behavior research methods, 52(5), 2232-2255. Ho, Ming-Chou, \& Atchley, P. (2008). Does the Object-Based Attention Effect Reflect a Benefit or a Cost? Chinese Journal of Psychology, 50(4), 347-356.

Kimchi, R., Yeshurun, Y., \& Cohen-Savransky, A. (2007). Automatic, stimulus-driven attentional capture by objecthood. Psychonomic Bulletin \& Review, 14(1), 166-172. 
Kret, M. E., \& Sjak-Shie, E. E. (2019). Preprocessing pupil size data: Guidelines and code. Behavior Research Methods, 51(3), 1336-1342.

Laeng, B., \& Endestad, T. (2012). Bright illusions reduce the eye's pupil. Proceedings of the National Academy of Sciences, 109(6), 2162-2167.

Lamy, D., \& Egeth, H. (2002). Object-based selection: The role of attentional shifts.

Perception \& Psychophysics, 64(1), 52-66. https://doi.org/10.3758/BF03194557

Loewenfeld, I. (1999). The pupil: Anatomy, physiology and clinical applications (Vol. 1). Boston.

Luo, C., \& Proctor, R. W. (2016). Perceptual grouping of objects occupied by target and flankers affects target-flanker interference. Attention, Perception, \& Psychophysics, 78(1), 251-263. https://doi.org/10.3758/s13414-015-0986-2

Mathôt, S., Dalmaijer, E., Grainger, J., \& Van der Stigchel, S. (2014). The pupillary light response reflects exogenous attention and inhibition of return. Journal of Vision, 14(14), $1-9$.

Mathôt, S., Van der Linden, L., Grainger, J., \& Vitu, F. (2013). The pupillary light response reveals the focus of covert visual attention. PloS One, 8(10), e78168.

Naber, M., Alvarez, G. A., \& Nakayama, K. (2013). Tracking the allocation of attention using human pupillary oscillations. Frontiers in Psychology, 4, 1-12.

Naber, M., \& Nakayama, K. (2013). Pupil responses to high-level image content. Journal of Vision, 13(6), 1-8.

Nah, J. C., \& Shomstein, S. (2020). Target frequency modulates object-based attention. Psychonomic Bulletin \& Review, 27, 981-989. 
Peirce, J., Gray, J. R., Simpson, S., MacAskill, M., Höchenberger, R., Sogo, H., Kastman, E., \& Lindeløv, J. K. (2019). PsychoPy2: Experiments in behavior made easy. Behavior Research Methods, 51(1), 195-203.

Posner, M. I. (1980). Orienting of attention. Quarterly Journal of Experimental Psychology, 32(1), 3-25.

R Core Team (2020). R: A language and environment for statistical computing. R Foundation for

Statistical Computing, Vienna, Austria. URL https://www.R-project.org/.

Reppa, I., Schmidt, W. C., \& Leek, E. C. (2012). Successes and failures in producing attentional object-based cueing effects. Attention, Perception, \& Psychophysics, 74(1), 43-69.

Richard, A. M., Lee, H., \& Vecera, S. P. (2008). Attentional spreading in object-based attention. Journal of Experimental Psychology: Human Perception and Performance, 34(4), 842-853.

Schmidtke, J. (2018). Pupillometry in linguistic research: An introduction and review for second language researchers. Studies in Second Language Acquisition, 40(3), 529549.

Shomstein, S., \& Yantis, S. (2002). Object-based attention: Sensory modulation or priority setting? Perception \& psychophysics, 64(1), 41-51.

Tkacz-Domb, S., \& Yeshurun, Y. (2018). The size of the attentional window when measured by the pupillary response to light. Scientific Reports, 8(1), 1-7. 
Wolff, M. J., Scholz, S., Akyürek, E. G., \& van Rijn, H. (2015). Two visual targets for the price of one? Pupil dilation shows reduced mental effort through temporal integration. Psychonomic Bulletin \& Review, 22(1), 251-257.

Yeshurun, Y. (2019). The spatial distribution of attention. Current Opinion in Psychology, 29, 76-81.

Yeshurun, Y., \& Rashal, E. (2017). The typical advantage of object-based attention reflects reduced spatial cost. Journal of Experimental Psychology: Human Perception and Performance, 43(1), 69-77.

Zekveld, A. A., \& Kramer, S. E. (2014). Cognitive processing load across a wide range of listening conditions: Insights from pupillometry. Psychophysiology, 51(3), 277-284. 